TITLE:

\title{
Spectral profile of atomic emission lines and effects of pulse duration on laser ablation in liquid
}

$\operatorname{AUTHOR}(S)$ :

Sakka, Tetsuo; Masai, Satoru; Fukami, Kazuhiro; Ogata, Yukio H.

\section{CITATION:}

Sakka, Tetsuo ... [et al]. Spectral profile of atomic emission lines and effects of pulse duration on laser ablation in liquid. Spectrochimica Acta Part B: Atomic Spectroscopy 2009, 64(10): 981-985

ISSUE DATE:

2009-10

URL:

http://hdl.handle.net/2433/91541

\section{RIGHT:}

c 2009 Elsevier B.V. All rights reserved.; この論文は出版社版でありませ ん。引用の際には出版社版をご確認ご利用ください。; This is not the published version. Please cite only the published version. 
Spectral profile of atomic emission lines and effects of pulse duration on laser ablation in liquid

\author{
Tetsuo Sakka ${ }^{1,2}$, Satoru Masai ${ }^{1}$, Kazuhiro Fukami ${ }^{1}$, Yukio H. Ogata ${ }^{1}$ \\ 1. Institute of Advanced Energy, Kyoto University, Uji, Kyoto 611-0011, Japan \\ 2. Research Fellow, Institute of Sustainability Science, Kyoto University, Uji, Kyoto \\ 611-0011, Japan
}

Corresponding author: Tetsuo Sakka

Institute of Advanced Energy, Kyoto University, Uji, Kyoto 611-0011, Japan

Phone: +81-774-38-3501, FAX: +81-774-38-3499, Email: t-sakka@iae.kyoto-u.ac.jp

\begin{abstract}
The emission spectra of laser-ablated $\mathrm{Cu}$ atoms in water were examined, focusing on the irradiation-pulse duration effects. Spectral line profile was observed for the pulse duration of 19, 90, and 150 ns at various delay times. The line width as narrow as instrumental width was obtained by 150 -ns pulse at the delay time of 800 ns. Also, long pulses result in high intensity of the emission. The spectral feature obtained by long pulses looks similar to that obtained in a gas phase. The absorption of the later part of the long pulse directly by the plume having been formed by the earlier part of the pulse may be the cause of this gas-phase-like emission. Whether the pulse heats directly the surface or the plume was investigated by the measurements of the removal volume of the ablation pit obtained by laser confocal scanning microscopy and the maximum bubble expansion size observed by shadowgraphy.
\end{abstract}

Key words: Underwater LIBS; Liquid-phase laser ablation; Shadowgraphy; Pulse-width effect; Ablation damage 


\section{Introduction}

Laser induced breakdown spectroscopy (LIBS) of solid surfaces submerged in liquid has great potential in many applications, such as elemental analysis of underwater sedimentation [1] or in situ analysis of electrodeposits [2], etc. However, if a usual ns pulse with the width of 10 ns or so is used as an ablation laser, the emission spectra from the plume in liquid give intense continuum $[3,4]$, which is not preferable for the application of LIBS to elemental analysis of solid surfaces submerged in liquid. To overcome this problem, double-pulse irradiation has been proposed [5,6]. Recently, we have published a preliminary result that the irradiation of a long nanosecond single pulse gives less-broadened emission spectral lines from the ablation plume in liquid environment without noticeable continuum from the background plasma [7]. Furthermore, the long ns pulse seems to give more intense emission than short pulses. According to these results irradiation with long ns pulses are promising in application to in situ LIBS in water, or maybe in any transparent liquid.

Also interesting is that surface damage could be surprisingly small if the pulse width of 150 ns was used as the ablation laser. This was suggested in our previous work, in which the ablation damage of electrodeposited $\mathrm{Cu}$ film in water was estimated from the number of pulses required to penetrate the film, when the pulse width of 150 ns was used as an ablation laser [8]. Low damage by the irradiation with a long ns pulse is also expected for sample surfaces much more solid than the electrodeposited film examined in our previous work.

The above findings can be summarized as follows: a long ns pulse gives intense emission, although the ablation efficiency is pretty low. This is an important advantage for the application to surface analysis. On the other hand, the mechanism of such improvement is not clear so far. To pursue this aspect of the phenomena further, it is important to clarify the mechanism of the laser ablation by a long ns pulse. The studies on 
the laser breakdown in bulk water also suggest the importance of the absorption of laser by the plasma $[9,10,11,12]$. Here we suppose that the most important aspect of the pulse-duration effect is the difference in the fraction of pulse energy directly deposited to the surface without being absorbed by the plume. Although we cannot directly measure this quantity, the size of the pit left after irradiation should be related to the energy given to the surface. Also, the maximum size of the cavity or bubble expansion after pulsed-laser breakdown is known to be proportional to the energy that the bubble has gained [13].

In the present paper we report on $\sim 0.2$-nm-resolution emission spectra of the plume for various pulse widths of ablation laser, namely, 19, 90 or 150 ns. The line profiles were also examined for various delay times. To have information on the energy deposited to surface, the ablation efficiency was studied in detail by examining the volume of the pit left on a Cu target. Also, to have additional information on the energy deposited to the plume, the plume expansion size or the maximum cavity size was compared for different pulse widths on the basis of the shadowgraphy of cavity expansion.

\section{Experimental}

The experimental setup for the measurement of emission spectra is basically the same as our previous studies and given elsewhere [2,7]. Briefly, a home-build Q-switched Nd:YAG laser was used as an ablation laser. The laser pulse width employed in the present work was 19,90 , or 150 ns. The pulse width was measured as a full width at half maximum of the pulse profile obtained by a fast silicon-PIN photodiode. The laser beam size was $4 \mathrm{~mm}$ in diameter and was multimode. The laser beam was focused onto a $\mathrm{Cu}$ plate immersed in water. A cubic quartz cell with the sides being $40 \mathrm{~mm}$ long was 
used for lateral observation of the optical emission from the ablation plume. The emitted light was collected by a lens system and focused onto an entrance slit of the 1-m-focal-length spectrograph (Ritsu Oyo Kogaku, MC100N) equipped with an intensified charge-coupled device (ICCD, Princeton Instruments, ICCD-1024MTDGE/1) as a detector. Since the entrance slit was set to $150 \mu \mathrm{m}$ to have good signal-to-noise ratio, the resolution of the system was $\sim 0.2 \mathrm{~nm}$. Timing of the data acquisition was controlled by the gating operation of the ICCD by a pulser (Princeton Instruments, PG200).

Figure 1 shows a schematic illustration of the experimental setup for shadowgraph measurement. The same laser, focusing optics, target, and cell, as used in emission spectroscopy, were used. The laser-ablation region was illuminated from the back-side by a high intensity Xe lamp (Ushio, SX-UI500XQ), and photographed by the ICCD camera. A cavity or a bubble produced on the target blocks the back-side illumination, so that we can obtained a shadow image of the cavity or the bubble. The shadowgraph was obtained at various time delays for each pulse width. The time resolution of the measurement was attained by gating operation of the ICCD. The gate width was 400 ns for the measurements with the delay of 400 ns and 1500 ns, while the gate width of $2 \mu$ s was employed for the measurements with the delay of $100 \mu$ s or longer.

The size of the pit formed by ablation was systematically measured. Again, $\mathrm{Cu}$ was employed as a target. Confocal laser scanning microscopy was employed to observe 3-dimensional image of the pits and the volume of the material removal was calculated by the software installed in the confocal laser scanning microscope system (Olympus, OLS1000). The volume removal was measured as a function of the laser pulse shot number.

\section{Results}


The emission spectra of the ablation plume obtained by the irradiation of a $\mathrm{Cu}$ target in water are given in Fig. 2 for various pulse widths. The spectral lines observed in this spectral range is assigned to the $3 \mathrm{~d}^{10} 4 \mathrm{~s}^{12} \mathrm{~S}-3 \mathrm{~d}^{10} 4 \mathrm{p}^{12} \mathrm{P}^{0}$ transition of atomic $\mathrm{Cu}$ [14]. Note that the lower state has a configuration of the ground state, i. e., [Ar core] $+3 d^{10} 4 s^{1}$. The upper state has a configuration of [Ar core] $+3 \mathrm{~d}^{10} 4 \mathrm{p}^{1}$. This configuration has a fine structure, i. e., a doublet, ${ }^{2} \mathrm{P}^{\mathrm{o}}{ }_{3 / 2}$ and ${ }^{2} \mathrm{P}^{\mathrm{o}}{ }_{1 / 2}$, with a splitting of $248 \mathrm{~cm}^{-1}$. Therefore, two transition lines appear, at $324.8 \mathrm{~nm}\left({ }^{2} \mathrm{~S}_{1 / 2}-{ }^{2} \mathrm{P}^{0}{ }_{3 / 2}\right)$ and $327.4 \mathrm{~nm}\left({ }^{2} \mathrm{~S}_{1 / 2}-{ }^{2} \mathrm{P}^{0}{ }_{1 / 2}\right)$. These lines were expected to give strong self-absorption, because of the high population of the lower state, which is the ground state. We selected these lines to demonstrate clearly the effects of the pulse width and the advantage of the long pulse. For all the delay times employed in the measurement, the intensity of emission was high enough to evaluate the spectral line width when the pulse width of 90 ns or 150 ns was employed, while we could not obtain clear spectral feature by the irradiation with a 19-ns pulse. This agrees with our previous work based on lower-resolution spectral measurements [7]. The spectral width of the line depends on the delay time. We obtained the narrowest width of $0.18 \mathrm{~nm}$ at the delay time of $800 \mathrm{~ns}$, which corresponds to the instrumental broadening of the spectrograph-ICCD system employed in the experiment. Also, we observed self-reversed absorption in the middle of the emission line spectral feature in the case of 90 ns pulse width for all the delay times employed in the measurement and also for150-ns pulse for the delay time of 500 ns. The results suggest that the characteristics of the plumes are sensitive to the laser pulse width.

Figure 3 (a) and (b) show the volume removed by laser ablation of $\mathrm{Cu}$ in air and in water, respectively, as a function of the number of pulse shots. The results give the same tendency for all the pulse shot numbers employed, especially it is clearly seen for 5 , 10, and 25 shots. This suggests reproducibility of the results, if the linearity of data points is assumed. In the case of the ablation in water (Fig. 3(b)), the ablation efficiency is drastically reduced by the use of a long pulse, such as 90 or 150 ns. The volume removal 
for 150 ns was negligibly small, although the irradiation spot was roughened by the ablation event. In air, the pulse width effect is not as clear as in water, i.e., the volume removal shows the same order for three pulse widths examined in the experiment. The irradiation by 19-ns pulse gives the volume removal significantly larger in water than in air, as has been observed in previous studies [8,15], while for the longer pulses the volume removal in water is rather smaller than in air.

Figure 4 shows shadowgraph images obtained with the pulse width of 19, 90, and 150 ns. For each pulse width shadowgraph was taken at various delay times to clarify the time evolution. In the beginning, namely $400 \mathrm{~ns}$ from the irradiation, we observed bright emission from the plume for 90- and 150-ns pulses. It is clear that long ns pulses give intense emission. This is consistent with the results of the emission spectra, showing high intensity for long ns pulses. Unfortunately, due to the bright emission we could not clarify if the cavity or the bubble starts to grow at this time for the long ns pulses, while a small bubble-like feature is seen in the shadowgraph taken by 19-ns-pulse irradiation. After the extinction of the emission, the bubble growth is clearly seen in the time range of $1 \mu$ s to $100 \mu$ s. The bubble expands to its maximum size at 100 $\mu$ s, and then it shrinks its size and collapses. The bubble size measured by a height of the hemisphere gives a maximum of $\sim 1 \mathrm{~mm}$ before collapse. We could not observe a significant difference between the results for the pulse widths of 90 and 150 ns, while the pulse width of 19 ns seems to give smaller sizes in all the delay times of the observation. Note that we can only take one shadowgraph image by a single pulse irradiation, which means that the tendency to obtain smaller size for the irradiation of a 19-ns pulse is reproduced by all the measurements for various delay times.

\section{Discussion}


It is clearly seen in Fig. 2 that the spectral measurements using a long ns pulse always give extremely narrow widths for the $\mathrm{Cu}$ atomic emission lines at $324.8 \mathrm{~nm}$ $\left({ }^{2} \mathrm{~S}_{1 / 2}-{ }^{2} \mathrm{P}^{\mathrm{o}}{ }_{3 / 2}\right)$ and $327.4 \mathrm{~nm}\left({ }^{2} \mathrm{~S}_{1 / 2}-{ }^{2} \mathrm{P}^{\mathrm{o}}{ }_{1 / 2}\right)$. The spectral line observed at the delay time of 800 ns by the 150-ns pulse gave the narrowest line among all the lines shown in Fig. 2. The width of this narrowest line is comparable to the instrumental width of the present experimental system. This is a promising result for the application to in situ LIBS. However, all the other spectral lines suffer from the broadening effect, i.e., Stark broadening, collision broadening or the broadening by the self-absorption effect [16]. The emission intensity observed by the 19-ns-pulse irradiation is very weak. This is due to a fast quenching of the plume in water [17]. If we use a higher fluence, the emission is clearly observed, although the spectra show an intense continuum.

Most of the lines in Fig. 2 show a self-reversed structure in the middle of the emission feature. The origin of the self-reversed structure is that emission from a high-temperature core region is absorbed by the surrounding low temperature region $[16,18,19,20]$. The temperature gradient seems to be more prominently influencing the spectra in water than in air. This is consistent with the fast quenching in water, which gives temperature decrease from a high temperature plume to room temperature water within a small thickness. Another condition that is necessary for this spectral feature to appear is that the emission region should be optically thick, as is often the case for laser-induced plasmas, or laser-ablation plumes. This means that the quantitative analysis based on the proportionality between the spectral-line intensity and the population density is quite difficult, at least, as long as we observe the self-reversed structure. In other words, the line intensity does not directly represent the population density, and hence, quantity of a certain element. We need a quite elaborate process for the quantitative estimation of the elemental density from a spectral line being influenced by self-absorption effects $[16,21,22]$. On the other hand, as seen in Fig. 2(b), the results obtained by a $150 \mathrm{~ns}$ pulse at the delay time of $800 \mathrm{~ns}$, the spectral lines are free from the 
self-reversed structure. The self-absorption might be less serious in this case. However, the spectra are still suffering from the self-absorption effects, as suggested by the intensity of the two lines, i.e., similar intensity for the fine-structured doublet lines are observed, while it should be 2:1 for 324.8 and $327.4 \mathrm{~nm}$ lines in the self-absorption-free spectra, since the former has twice as large statistical weight for its upper state as the latter line has. The self-absorption effect results in leveling of the intensity of these two lines.

Such a strong self-absorption effect is basically due to a high density of the light emitting region, while it is also due to a high population density of the lower state of the transition, i.e., the lower state is the ground state, the population of which is the highest. From the point of view of the Boltzmann distribution the ratio of the two levels involved in the transition is proportional to the Boltzmann factor. Since the self-absorption preferably occurs for the transition with a highly-populated lower level, the ground state has a high chance to show significant self-absorption effects.

The present results are, therefore, not satisfactory from the point of view of "free of self-absorption". However, the results given by long ns pulses show significant improvement, i.e., (a) spectral lines narrow enough to distinguish elements, (b) very little continuum in any delay times later than 500 ns, (c) spectral feature without an apparent self-reversal structure at delay times later than $800 \mathrm{~ns}$, and (d) extremely intense emission compared with the irradiation by a 20 ns pulse. These characteristics are all suitable for the application to LIBS measurement.

To improve the spectral feature further and enable quantitative analysis, the mechanism of line narrowing by the elongation of the pulse should be clarified. We here suppose that the plume can receive further energy directly from the later part of the long pulse. This means that we can excite the plume efficiently without depositing excess energy to the surface, avoiding an unnecessary damage to the surface. 
According to the results shown in Fig. 3(b), the damage to the sample surface seems to be minimized by using a long ns pulse, although the long pulse gives intense spectral lines. This behavior is opposite of the results obtained in air atmosphere, where ablation by a long pulse is more efficient than a short pulse (Fig. 3(a)). Also, it can be seen in the figure that ablation efficiency is higher in water than in air when a short pulse is used, while it is opposite when a long pulse is used. The ablation by a long pulse for a target in water gives lowest efficiency compared with any other irradiation conditions examined in Fig 3. When a long pulse is used for the target in water, the ablation pit volume is so small that it is comparable to the error in the pit volume measurement caused by the roughness of the surface due to the laser irradiation. The low damage observed for a long ns pulse seems to be inconsistent with the intense light emission, if we assume that the ablated species equally emit light with the same emission efficiency. The high light-emission efficiency for long-pulse irradiation with the ablation rate being quite low is rather explained by the rate of extinction in the plume, i.e., the extinction is usually quite fast for a small plume due to the presence of nearby water, while even a small increase in the plume size can lower the extinction rate, and can cause significant increase in the intensity of the emission. The present results are rather consistently explained if we assume that the later part of the long ns pulse is directly absorbed by the plume and does not reach to the surface. This is consistent to our recent work on the pulse-width effects upon the image of the plume [23], i.e., a long ns pulse gives a larger and clearer hemispherical plume, while a shorter pulse gives a smaller and rather flat emission image.

In line with this explanation, the maximum sizes of the bubble expansion observed in shadowgraph images can also be explained. According to a simple hydrodynamic calculation, the maximum bubble size is proportional to the energy given to the bubble [13]. Also, suppose that the bubble has taken in the excited region or the plume during its expansion. Then the highly excited plume should result in a larger bubble. Note that a large part of the energy deposited into the target diffuse into the bulk 
of the target and does not used to heat up the bubble, while the energy directly given to the plume ends up heating the bubble. The results of the maximum bubble size observed in the shadowgraph images is rather consistent to above discussion, i.e., a long ns pulse tends to give energy directly to the plume while a short pulse give energy to the surface.

\section{Conclusion}

Less-broadened spectral lines and a weak continuum in the emission spectra of the plume in liquid by a long ns laser pulse were examined, with special attention on the effect of pulse width. For the $3 d^{10} 4 s^{12} \mathrm{~S}-3 d^{10} 4 p^{12} \mathrm{P}^{0}$ transitions of atomic Cu observed at 324.8 and $327.4 \mathrm{~nm}$, the line width (FWHM) as narrow as $0.18 \mathrm{~nm}$ was observed by 150 -ns pulse at the delay time of 800 ns.

The pulse duration effect was assumed to be due to the absorption of the later part of the long pulse directly by the plume and the energy deposited to the surface is less for a long pulse than a short pulse. The size of the target damaging measured by laser confocal scanning microscopy and the maximum bubble size measured by shadowgraphy suggest low damaging and efficient heating of the plume by the use of longer pulses.

The long ns pulse is favorable for the application to in situ LIBS in liquid in the following reasons; a relatively slow heating of the plume causes a larger and less-dense plume, and therefore, the emission spectral lines can be less broadened with weak continuum, the emission can be fairly intense, and the surface damage is minimized.

\section{Acknowledgment}


This work was financially supported by a grant-in-aid from the Japan Society for the Promotion of Science. 
References

[1] V. Lazic, F. Colao, R. Fantoni, V. Spizzichino, S. Jovicevic, Underwater sediment analyses by laser induced breakdown spectroscopy and calibration procedure for fluctuating plasma parameters, Spectrochim. Acta B, 62 (2007) 30-39.

[2] T. Nishi, T. Sakka, H. Oguchi, K. Fukami, Y. H. Ogata, In situ electrode surface analysis by laser-induced breakdown spectroscopy, J. Electrochem. Soc., 155 (2008) F237-F240.

[3] T. Sakka, S. Iwanaga, Y. H. Ogata, A. Matsunawa, T. Takemoto, Laser ablation at solid-liquid interfaces: An approach from optical emission spectra, J. Chem. Phys. 112 (2000) 8645-8653.

[4] H. Ushida, N. Takada, K. Sasaki, Diagnostics of liquid-phase laser ablation plasmas by spectroscopic methods, J. Phys: Conf. Ser. 59 (2007) 563-566.

[5] A. E. Pichahchy, D. A. Cremers, M. J. Ferris, Elemental analysis of metals under water using laser-induced breakdown spectroscopy, Spectrochim. Acta B 52 (1997) 25-39.

[6] V. Lazic, F. Colao, R. Fantoni, V. Spizzicchino, Laser-induced breakdown spectroscopy in water: Improvement of the detection threshold by signal processing, Spectrochim. Acta B 60 (2005) 1002-10013. 
[7] T. Sakka, H. Oguchi, S. Masai, K. Hirata, Y. H. Ogata, M. Saeki, H. Ohba, Use of a long-duration ns pulse for efficient emission of spectral lines from the laser ablation plume in water, Appl. Phys. Lett. 88 (2006) 061120.

[8] T. Sakka, H. Oguchi, S. Masai, Y. H. Ogata, Quasi nondestructive elemental analysis of solid surface in liquid by long-pulse laser ablation plume spectroscopy, Chem. Lett. 36 (2007) 508-509.

[9] J. Noack, A. Vogel, Laser-Induced Plasma Formation in water at nanosecond to femtosecond time scales: Calculation of thresholds, absorption coefficients, and energy density, IEEE J. Quantum Electron., 35 (1999) 1156-1167.

[10] D. X. Hammer, E. D. Jansen, M. Frenz, G. D. Noojin, R. J. Thomas, J. Noack, A. Vogel, B. A. Rockwell, A. J. Welch, Shielding properties of laser-induced breakdown in water for pulse durations from 5 ns to 125 fs, Appl. Opt. 36 (1997) 5630-5640.

[11] A. Vogel, K. Nahen, D. Theisen, J. Noack, Plasma formation in water by picosecond and nanosecond Nd:YAG laser pulses-Part I: Optical breakdown at threshold and superthreshold irradiance, IEEE J. Select. Topics Quantum Electron., 2 (1996) 847-860.

[12] K. Nahen, A. Vogel, Plasma formation in water by picosecond and nanosecond Nd:YAG laser pulses-Part II: Transmission, scattering, and reflection, IEEE J. Select. Topics Quantum Electron., 2 (1996) 861-871.

[13] A. Vogel, S. Busch, U. Parlitz, Shock wave emission and cavitation bubble generation by picosecond and nanosecond optical breakdown in water, J. Acoust. Soc. Am., 100 (1996) 148-165. 
[14] P. L. Smith, C. Heise, J. R. Esmond, R. L. Kurucz, Kurucz Atomic Line Database, 1995 Atomic Line Data (R.L. Kurucz and B. Bell) Kurucz CD-ROM No. 23, http://www.cfa.harvard.edu/amp/ampdata/kurucz23/sekur.html

[15] S. Zhu, Y. F. Lu, M. H. Hong, X. Y. Chen, Laser ablation of solid substrates in water and ambient air, J. Appl. Phys., 89 (2001) 2400-2403.

[16] T. Sakka, T. Nakajima, Y. H. Ogata, Spatial population distribution of laser ablation species determined by self-reversed emission line profile, J. Appl. Phys. 92 (2002) 2296-2303.

[17] C. Haisch, J. Liermann, U. Panne, R. Niessner, Characterization of colloidal particles by laser-induced plasma spectroscopy (LIPS), Anal. Chim. Acta. 346 (1997) 23-35.

[18] R. D. Cowan, G. H. Dieke, Self-absorption of spectrum lines, Rev. Mod. Phys. 20 (1948) 418-455.

[19] J. Hermann, C. Boulmer-Leborgne, D. Hong, Diagnostics of the early phase of an ultraviolet laser induced plasma by spectral line analysis considering self-absorption, J. Appl. Phys., 83 (1998) 691-696.

[20] T. Sakka, K. Hirata, S. Masai, Y. H. Ogata, Spatial distribution of the species laser-ablated from the target submerged in liquid, Proc. SPIE, 5713 (2005) 276-284. 
[21] H. Furusawa, T. Sakka, Y. H. Ogata, J. Appl. Phys., Characterization of ablated species in laser-induced plasma plume, 96 (2004) 975-982.

[22] H. Furusawa, T. Sakka, Y. H. Ogata, Characterization of laser-induced plasma plume: Comparison between $\mathrm{Al}$ and $\mathrm{Al}_{2} \mathrm{O}_{3}$ targets, Appl. Phys. A79 (2004) 1291-1294.

[23] H. Oguchi, T. Sakka, Y. H. Ogata, Effects of pulse duration upon the plume formation by the laser ablation of Cu in water, J. Appl. Phys., 102 (2007) 023306. 
Figure captions

Fig. 1. Experimental setup for shadowgraph measurements.

Fig. 2. Emission spectra of $\mathrm{Cu}$ atoms in the ablation plume in water at the delay time of (a) 400 ns, (b) 800 ns, and 1000 ns after the laser irradiation. Pulse widths of 19, 90, and (c) 150 ns were compared. The laser pulse with the pulse energy of $1.7 \mathrm{~mJ}$ was used. The laser beam was focused onto the target by a 32.7-mm focal-length lens.

Fig. 3. Volume of the ablation pit left on the target as a function of pulse shot number (a) in air and (b) in water. Pulse widths of 19, 90, and 150 ns were compared. The irradiation was performed by laser pulse with the pulse energy of $10.7 \mathrm{~mJ}$, focused onto the target by a 70-mm focal-length lens.

Fig. 4. Shadowgraph of the bubble produced at the laser irradiation spot on a Cu target in water at various delay times from the irradiation. Pulse widths of 19, 90, and 150 ns were compared. The laser pulse with the pulse energy of $1.7 \mathrm{~mJ}$ was used for ablation. The laser beam was focused onto the target by a 32.7-mm focal-length lens. 


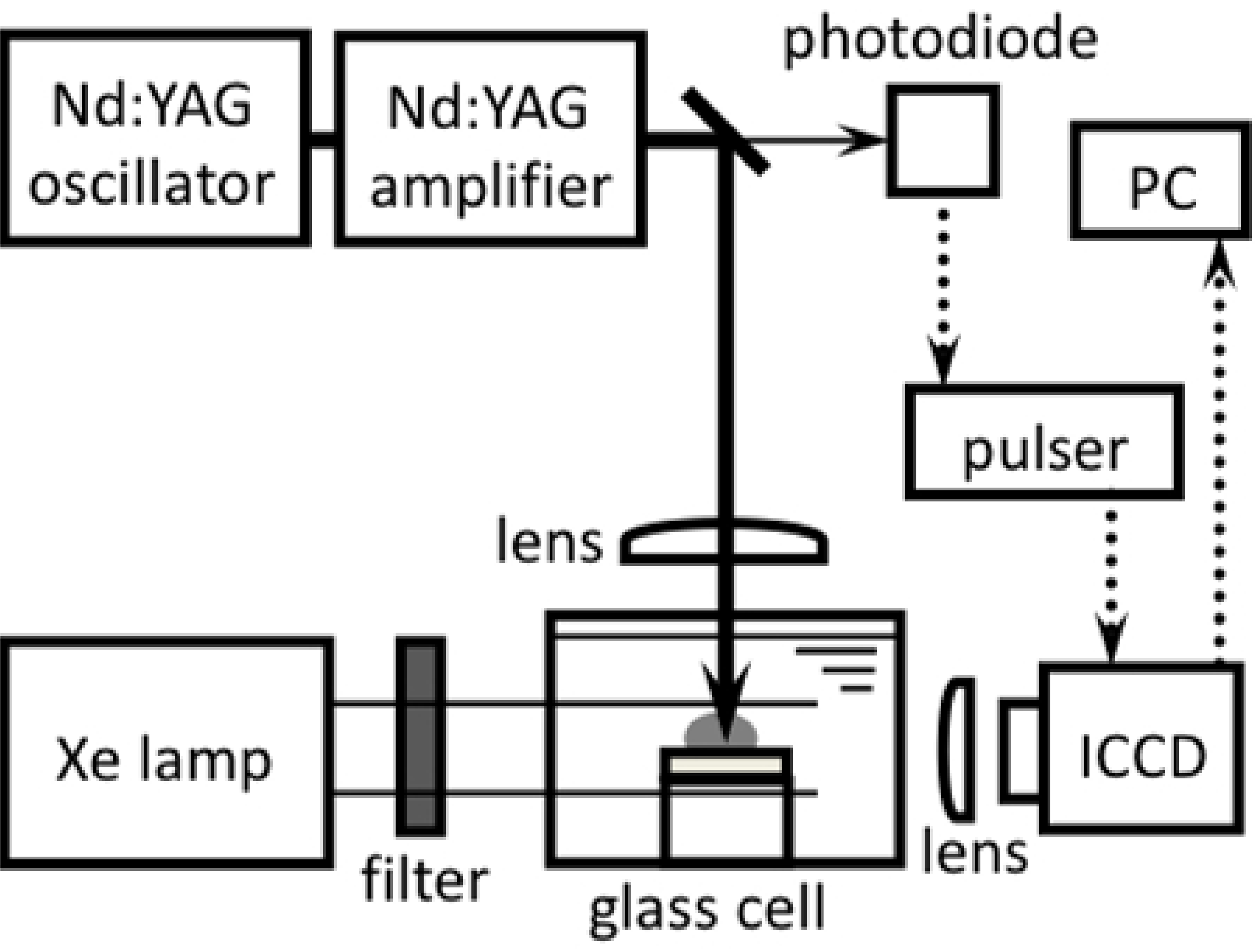


(a) delay $500 \mathrm{~ns}$

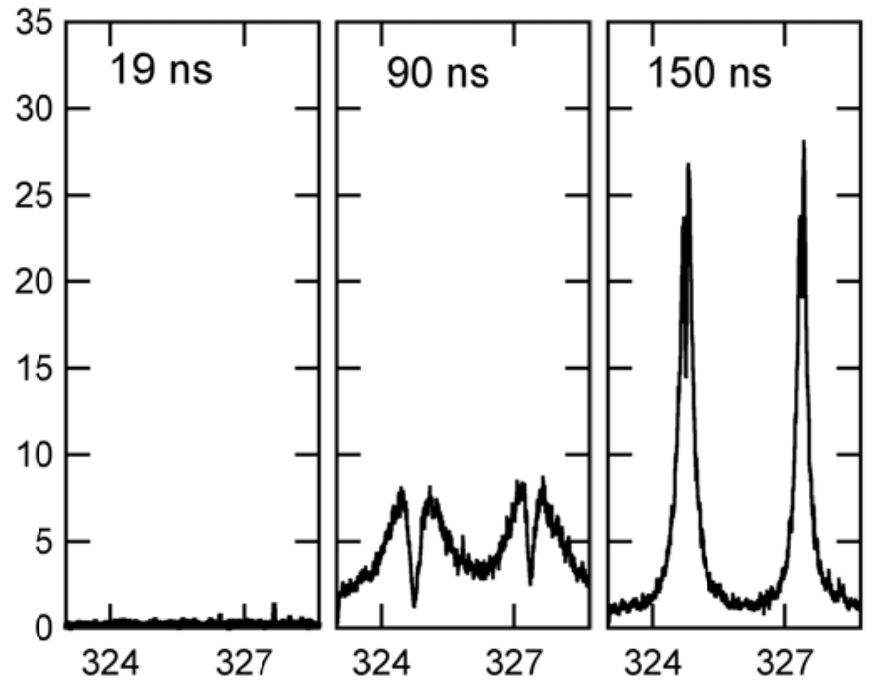

(b) delay $800 \mathrm{~ns}$

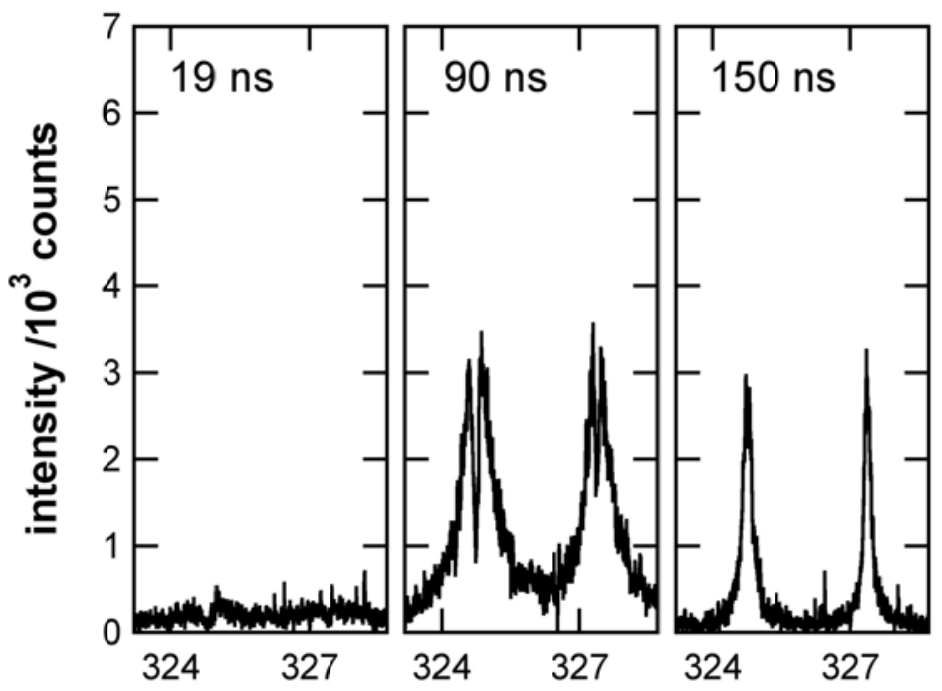

(c) delay $1000 \mathrm{~ns}$

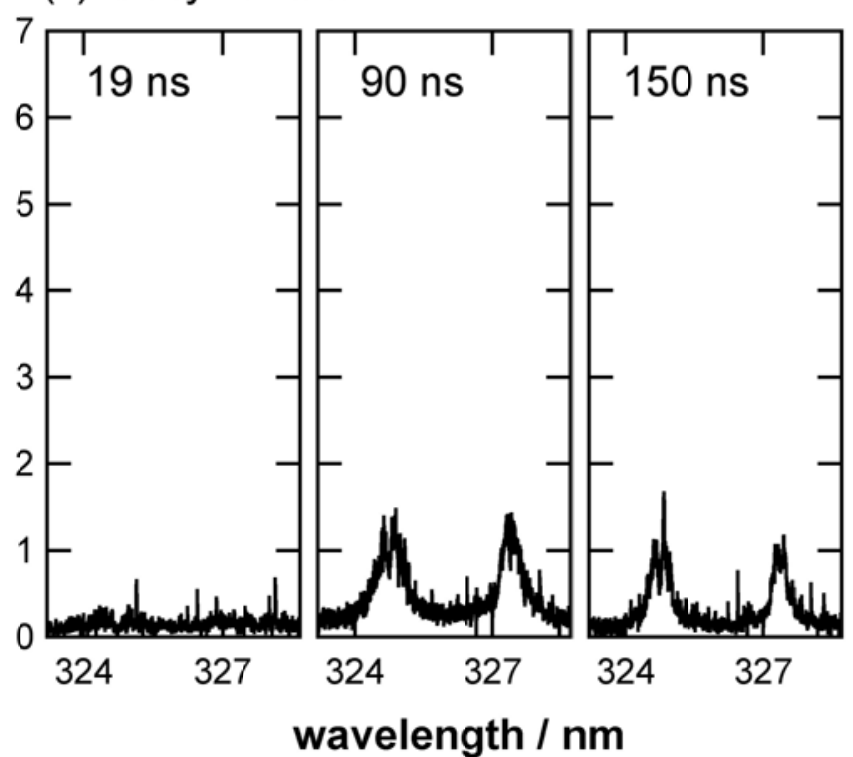




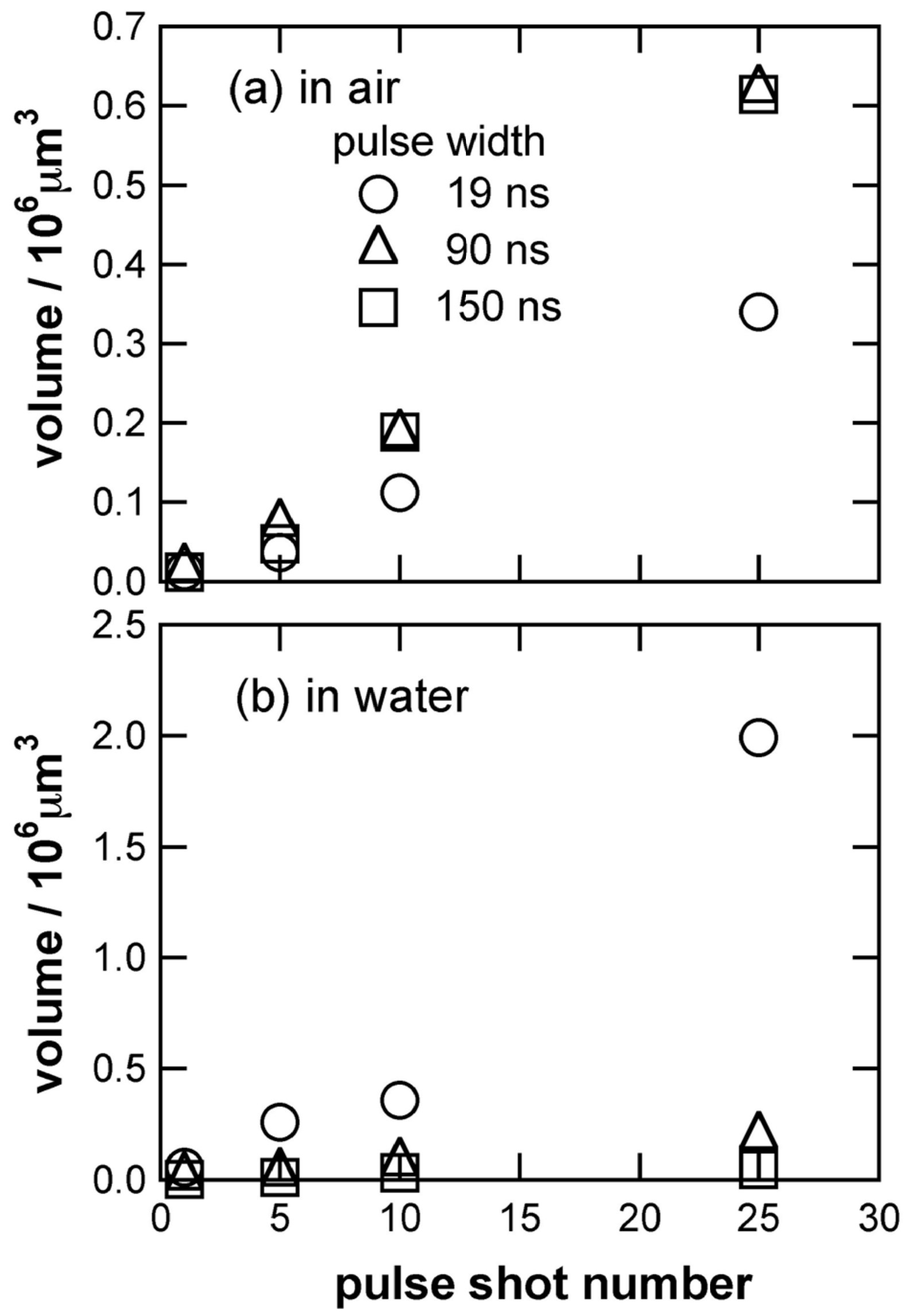


pulse width $=19 \mathrm{~ns}$
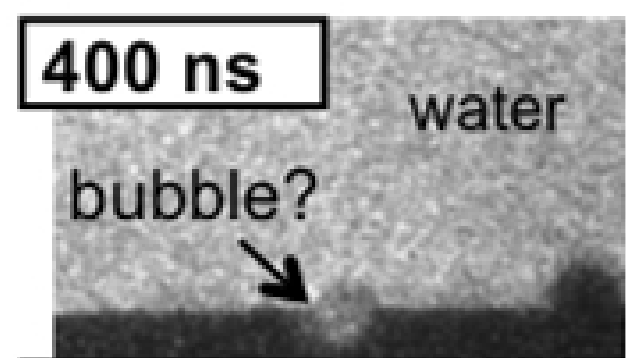

\section{$1500 \mathrm{~ns}$}

\section{$100 \mu \mathrm{s}$}

$150 \mu \mathrm{s}$
$90 \mathrm{~ns}$
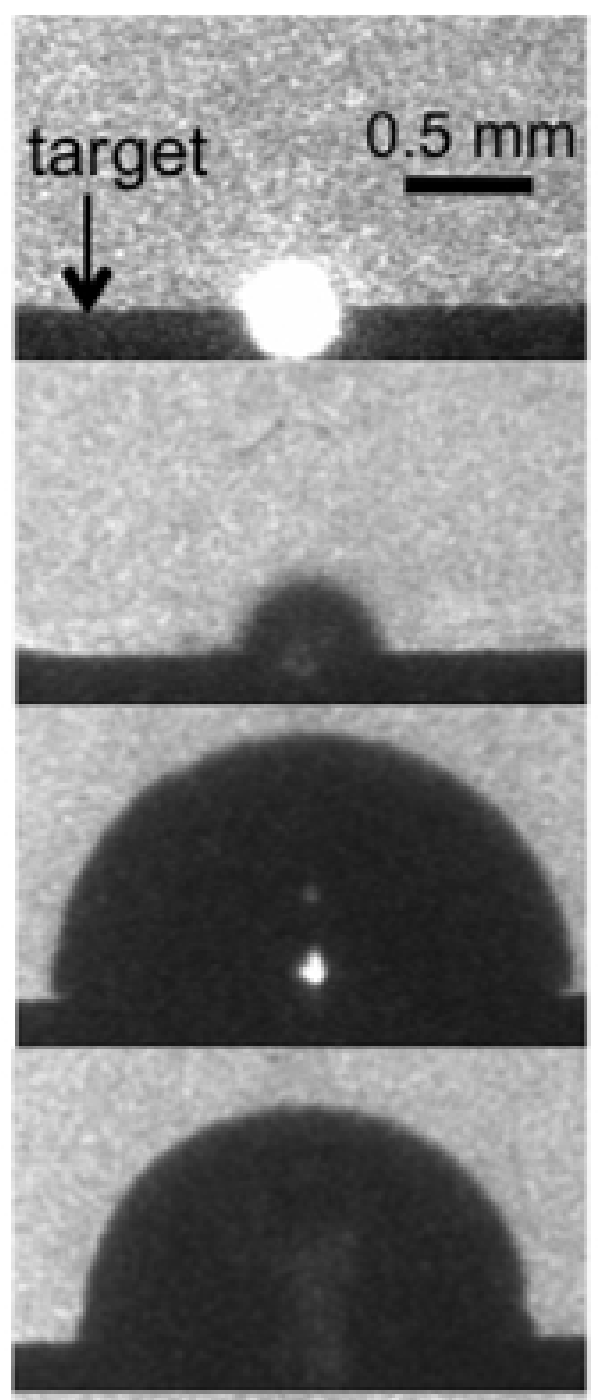

$200 \mu \mathrm{s}$

$400 \mu \mathrm{s}$

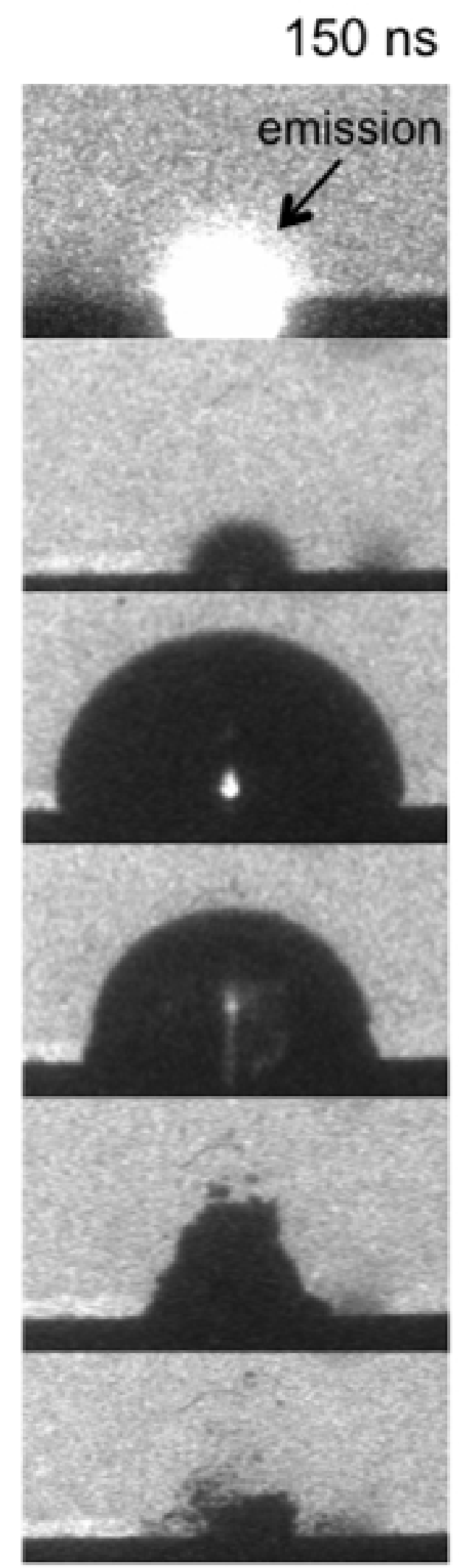

